\title{
Proton Resonance Frequency Chemical Shift Thermometry: Experimental Design and Validation toward High-Resolution Noninvasive Temperature Monitoring and In Vivo Experience in a Nonhuman Primate Model of Acute Ischemic Stroke
}

\author{
S. Dehkharghani, H. Mao, L. Howell, X. Zhang, K.S. Pate, P.R. Magrath, F. Tong, L. Wei, D. Qiu, C. Fleischer, and J.N. Oshinski
}

\begin{abstract}
BACKGROUND AND PURPOSE: Applications for noninvasive biologic temperature monitoring are widespread in biomedicine and of particular interest in the context of brain temperature regulation, where traditionally costly and invasive monitoring schemes limit their applicability in many settings. Brain thermal regulation, therefore, remains controversial, motivating the development of noninvasive approaches such as temperature-sensitive nuclear MR phenomena. The purpose of this work was to compare the utility of competing approaches to MR thermometry by using proton resonance frequency chemical shift. We tested 3 methodologies, hypothesizing the feasibility of a fast and accurate approach to chemical shift thermometry, in a phantom study at $3 \mathrm{~T}$.
\end{abstract}

MATERIALS AND METHODS: A conventional, paired approach (difference [DIFF]-1), an accelerated single-scan approach (DIFF-2), and a new, further accelerated strategy (DIFF-3) were tested. Phantom temperatures were modulated during real-time fiber optic temperature monitoring, with MR thermometry derived simultaneously from temperature-sensitive changes in the water proton chemical shift ( $\sim 0.01$ $\left.\mathrm{ppm} /{ }^{\circ} \mathrm{C}\right) . \mathrm{MR}$ thermometry was subsequently performed in a series of in vivo nonhuman primate experiments under physiologic and ischemic conditions, testing its reproducibility and overall performance.

RESULTS: Chemical shift thermometry demonstrated excellent agreement with phantom temperatures for all 3 approaches (DIFF-1: linear regression $R^{2}=0.994 ; P<.001$; acquisition time $=4$ minutes 40 seconds; DIFF- $2: R^{2}=0.996 ; P<.001$; acquisition time $=4$ minutes; DIFF-3: $R^{2}=0.998 ; P<.001$; acquisition time $=40$ seconds).

CONCLUSIONS: These findings confirm the comparability in performance of 3 competing approaches to MR thermometry and present in vivo applications under physiologic and ischemic conditions in a primate stroke model.

ABBREVIATIONS: DIFF = difference; $P R F=$ proton resonance frequencies

B rain temperature regulation is a poorly understood physiologic process at the intersection of complex metabolic and hemodynamic phenomena. ${ }^{1-5}$ The importance of temperature dysregulation as a driving force in various mechanisms of neuronal injury has been highlighted in past reports, and the exquisite

Received September 23, 2014; accepted after revision November 27.

From the Department of Radiology and Imaging Sciences (S.D., H.M., K.S.P., F.T., D.Q., J.N.O.), Emory University Hospital, Atlanta, Georgia; Yerkes National Primate Research Center (L.H., X.Z.), Emory University, Atlanta, Georgia; Department of Biomedical Engineering (P.R.M.), Northwestern University, Evanston, Illinois; and Department of Biomedical Engineering (L.W., C.F.), Emory University-Georgia Institute of Technology, Atlanta, Georgia.

This work was supported by the American Society of Neuroradiology Scholar Award in Neuroradiology Research (2012-2013, 2013-2014) and an Emory University Research Committee (2013-2014) grant awarded to the lead author; in part the $\mathrm{Na}$ tional Center for Research Resources; and currently by the Office of Research Infrastructure Programs of the National Institutes of Health (P51RR000165, OD P51OD011132).

Portions of these data previously presented as oral scientific abstracts at: Annual Meeting of the American Society of Neuroradiology and the Foundation of the ASNR Symposium, May 10-23, 2013; San Diego, California.

sensitivity of neuronal substrate to even modest hypothermia is well-described. ${ }^{6-10}$ Direct brain temperature measurements, however, remain difficult, and reports are limited in number; specifically, the cost and invasiveness of implantable temperature probes limit their applicability in clinically relevant models for characterization of either physiologic or pathologic cerebral spatial temperature gradients. ${ }^{11-14}$ Similarly, systemic surrogates of brain temperature, such as tympanic or rectal probes, may be inaccurate as estimates of brain temperature, particularly during dysregulation, and furthermore unable to address the presence of spatial cerebral temperature gradients directly. ${ }^{15-19}$

Noninvasive imaging approaches to brain temperature monitoring have thus been explored. ${ }^{20}$ Among these, the use of MR

Please address correspondence to Seena Dehkharghani, MD, Neuroradiology Division, Attention: Mary Davis, Department of Radiology and Imaging Sciences, Emory University Hospital, 1364 Clifton Rd, NE, Atlanta, GA 30322; e-mail: seena.dehkharghani@emory.edu

- Indicates open access to non-subscribers at www.ajnr.org

http://dx.doi.org/10.3174/ajnr.A4241 
spectroscopy to measure temperature-dependent fluctuations in the relative difference between proton resonance frequencies (PRF), such as the chemical shift difference of water and $N$-acetylaspartate, has gained interest for its theoretic ability to generate quantitative cerebral temperature maps as demonstrated in recent human cerebral thermometry by Karaszewski et al $^{21-24}$ and Marshall et al. ${ }^{25}$ Briefly, the PRF method of thermometry is predicated on the identification of a static, non-temperature-dependent resonance frequency (eg, the methyl resonance of NAA at approximately $2.01 \mathrm{ppm}$ ) to serve as an internal reference for the temperature-sensitive water proton resonance (nominally assigned at 4.7 ppm), which, by comparison, exhibits frequency shifts due to changes in hydrogen bonding equilibrium and electron shielding. ${ }^{26}$ The robust presence of both NAA and water within brain tissues facilitates detection and measurement, making this technique practical as an approach to cerebral thermometry. Accurate determination of the chemical shift difference between the water and NAA resonance frequencies $\left(\Delta \delta_{\text {water-NAA }}\right)$ has been shown to correlate well with changes in temperature $\left(0.01 \mathrm{ppm} /{ }^{\circ} \mathrm{C}\right)$ and to correlate uniformly across physiologic ranges of $\mathrm{pH}$ and protein concentration. ${ }^{27}$ PRF thermometry may be acquired with single-voxel spectroscopy or the more time-consuming multivoxel techniques known commonly as chemical shift imaging or MR spectroscopic imaging. The latter, despite several limitations, has the potential for generation of chemical shift grids and maps for simultaneous measurement of temperature distribution across brain regions. ${ }^{26,28,29}$

Initial studies by Corbett et $\mathrm{al}^{27}$ used a paired acquisition strategy, consisting first of a non-water-suppressed proton spectrum, allowing the detection of the large water peak in the hydrogen PRF spectrum, followed by a water-suppressed spectrum, in which suppression/reduction of the water signal augments the detectability of lower amplitude hydrogen metabolites such as NAA. In doing so, the water-NAA chemical shift is measured from paired spectra, introducing potential errors related to interscan temperature changes or motion between the 2 acquisitions. It has been proposed that a single, partially water-suppressed spectrum may provide sufficient residual water signal for an "internally referenced" water-NAA chemical shift measurement, and subsequent studies adopted this strategy, albeit without report of direct experimental validation. ${ }^{15,30}$

Distortion of the water peak with water-suppression techniques could impart errors in center frequency estimation and thus impose pitfalls in such a single-scan approach to PRF thermometry; the rigorous and detailed direct comparison of competing approaches is therefore lacking. This work presents the comparative utility of competing approaches to PRF thermometry under reproducible experimental conditions, with primary attention to speed and accuracy by using a flow-modulated and temperature-controlled phantom and a clinical $3 \mathrm{~T}$ scanner. In vivo experience with single- and multivoxel MR thermometry is then presented in 2 nonhuman primate experiments during physiologic and ischemic conditions.

\section{MATERIALS AND METHODS \\ Phantom Model}

All imaging experiments for this study were performed on a 3T Tim Trio whole-body system (Siemens, Erlangen, Germany). The phantom model in use for this study consists of the following:
1) an aqueous brain cytosol spectroscopy phantom prepared in our laboratory with varying compositions of selected, known cerebral metabolites, including NAA, lactate, glutamate, choline, and creatine; 2) a circulating water bath created specifically for use in the following series of MR thermometry experiments; and 3) a 4-channel MR imaging-compatible fiber optic thermometry system. The design and validation of the circulating water bath are detailed subsequently.

For the purposes of the following thermometry calibration experiments, NAA (the nonwater metabolite of interest for PRF thermometry conducted herein) was maintained at approximately $20 \mathrm{mmol} / \mathrm{L}$. Paramagnetic agents (gadolinium) were used in the iterative calibration and optimization of relaxation times and resonance line widths.

An MR imaging-compatible, circulating water bath coupled to a temperature control system was built for temperature regulation in these experiments, modulated by a proportional-integral-derivative controller connected to a 1000 -W heating element and optimized to control brain cytosol phantom temperatures during ${ }^{1} \mathrm{H}$-spectroscopy experiments. The accuracy of the model and circulating water bath was tested before spectroscopy experiments; a proportional-integral-derivative input sequence steadily increasing phantom temperatures from approximately 18 to $45^{\circ} \mathrm{C}$ for 2 hours was prescribed. The phantom was then placed in the MR imaging scanner where actual reference temperatures were recorded during simultaneous MR spectroscopy. Additional system testing confirmed excellent stability and control of temperature over the prescribed range $\left( \pm 0.1^{\circ} \mathrm{C}\right)$.

Fiber optic thermometry was performed in real-time during all phantom experiments and was monitored by an MR imagingcompatible high-resolution $\left( \pm 0.01^{\circ} \mathrm{C}, 3\right.$-second temporal update) 4-channel bandgap gallium arsenide system (OTG-M series; OpSens, Quebec, Quebec, Canada).

\section{PRF Thermometry}

A series of experiments was undertaken to compare the existing and proposed approaches to PRF thermometry using $\Delta \delta_{\text {water-NAA }}$. The metabolite solution was secured in a bottle within the phantom and monitored at room temperature $\left(\sim 18-19^{\circ} \mathrm{C}\right)$ in the preliminary experience with the system. Despite the cold forced air within the scanner, only minimal fluctuations $\left(<0.3^{\circ} \mathrm{C}\right)$ in equilibrium temperatures were observed preceding thermometry experiments with simultaneous probe and MR spectroscopy thermometry acquired as detailed below.

Techniques. Three approaches to PRF thermometry were investigated, denoted hereafter as difference (DIFF) 1, DIFF 2, and DIFF 3 (Fig 1A). The initial experiment (DIFF 1) used a traditional paired-scan technique as described by Corbett et al. ${ }^{27}$ Briefly, pairs of single-voxel spectroscopy interleaving non-water-suppressed and water-suppressed spectra were sequentially acquired across modulated temperatures ranging between $\sim 18$ and $45^{\circ} \mathrm{C}$. These paired spectra were repeatedly acquired in succession, both by using a point-resolved spectroscopy sequence, and collected during approximately 140 minutes with real-time fiber optic temperature monitoring.

Past investigations have used an accelerated, single-scan prescription for PRF thermometry, and this approach (DIFF 2) was 

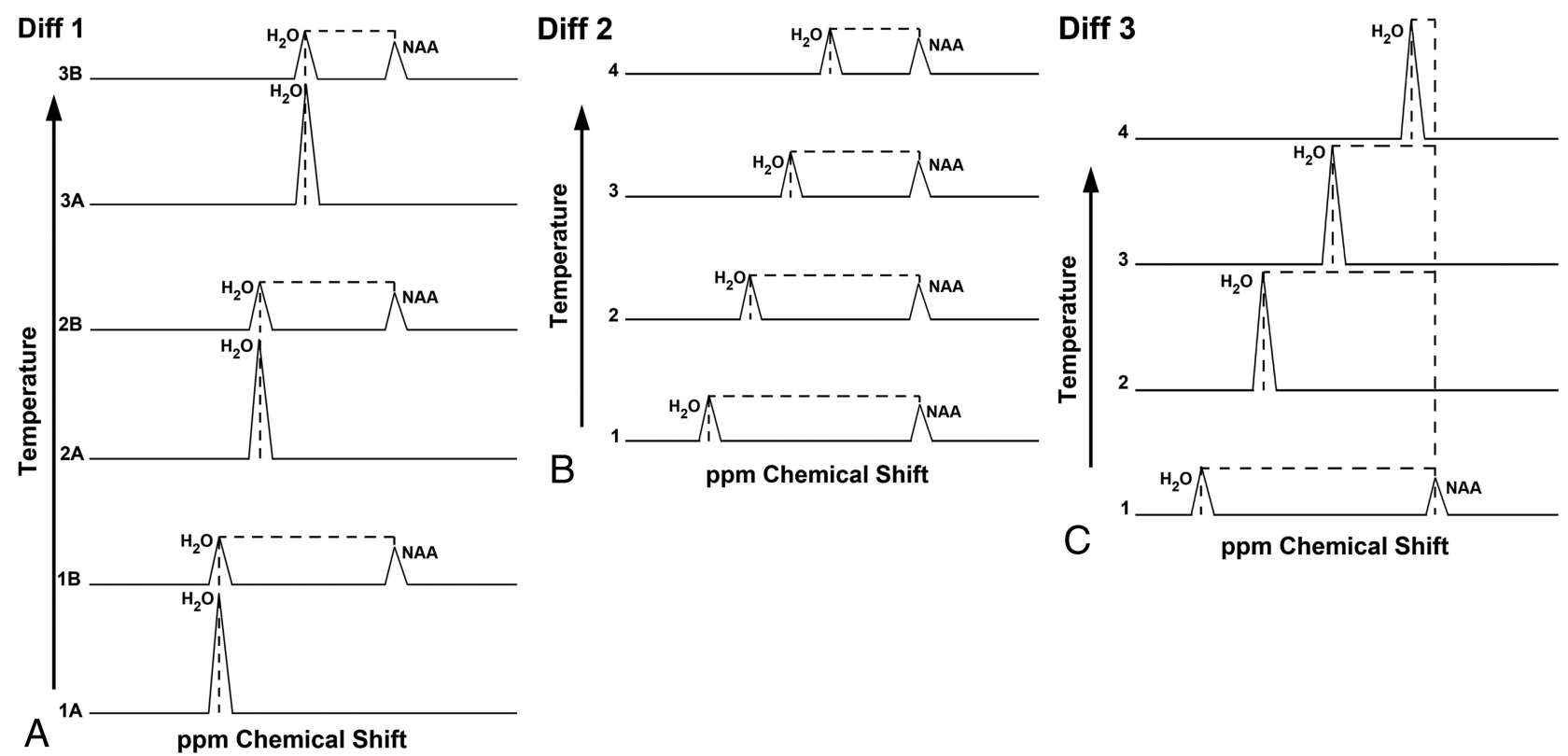

FIG 1. Schematic representation of methodologies for PRF thermometry: DIFF 1, DIFF 2, and DIFF 3 (see text). With increasing temperatures, the water resonance frequency is observed to shift at approximately $0.01 \mathrm{ppm} /{ }^{\circ} \mathrm{C}$ toward the lower resonance. A, DIFF 1 , representing the initial-approach interleaving pairs of non-water-suppressed and water-suppressed proton spectra during increasing temperatures. Alphanumeric combinations denote the pair number, followed by either A for non-water-suppressed or B for watersuppressed spectra. The shifting water-resonance frequency is thus measured against the static NAA resonance, the latter obtained from $B$ in each pair. B, DIFF 2 partially water-suppressed spectrum used for the simultaneous measurement of both water and NAA frequencies. $C$, DIFF 3, newly proposed accelerated methodology, wherein the NAA reference frequency is obtained from an initial water-suppressed spectrum and is followed by a high-temporal-resolution series of non-water-suppressed spectra, measured against the initial NAA reference frequency.

tested in a subsequent experiment $($ Fig $1 B) .{ }^{15}$ Specifically, given the stationary resonance of the methyl NAA reference frequency $(\sim 2.01 \mathrm{ppm})$, this approach uses only partial suppression of the water resonance, allowing simultaneous water and NAA peak assignments from a given spectrum, theoretically obviating the less time-efficient paired strategy in DIFF 1. Simultaneous assignment of both water and NAA peaks may therefore mitigate potential concerns over interscan motion or temperature changes inherent to the paired approach in DIFF 1. In this manner, DIFF 2 was measured and plotted against probe reference temperatures under otherwise similar experimental conditions as described in DIFF 1.

As an alternative to either preceding methodology, given our observation of stability in the NAA reference frequency as previously reported, we tested a new, third approach with further predicted improvements in temporal resolution. ${ }^{27}$ This hybridized approach (DIFF 3) was tested using a single initial NAA reference frequency, followed by a series of rapid ( $\sim 40$-second) non-watersuppressed metabolite scans, under conditions similar to those described in DIFF 1 and DIFF 2 with temperatures steadily increased to $35^{\circ} \mathrm{C}$ (Fig $1 C$ ).

MR Spectroscopy Postprocessing and Analysis. Scanner acquisition parameters were optimized for 3T imaging and designed to approximate conditions for future in vivo and clinical applications to the extent achievable. T1-weighted images were first obtained by using a T1 MPRAGE sequence $(\mathrm{TR}=2300 \mathrm{~ms}$, $\mathrm{TE}=$ $3.68 \mathrm{~ms}, \mathrm{TI}=900 \mathrm{~ms}$, flip angle $=9^{\circ}$, voxel size $=1 \times 1 \times 1 \mathrm{~mm}^{3}$ ) for localization. All spectra were acquired as follows: $8-\mathrm{mL}$ singlevoxel point-resolved spectroscopy sequence with $\mathrm{TR}=2$ seconds;
$\mathrm{TE}=30 \mathrm{~ms}$; signal averages $=16$ (without water suppression) and 64 (with water suppression), giving an acquisition time of 40 seconds (non-water-suppressed) and $\sim 4$ minutes (watersuppressed), respectively. Water suppression used for metabolite frequency assignments was achieved by a standard 3pulse chemical shift selective water suppression technique (35- to $50-\mathrm{Hz}$ suppression bandwidth). Center frequency was set on the water resonance initially. Following the advanced automated shimming protocol equipped on the scanner, manual shimming was applied to further refine the shimming conditions.

Spectroscopic data were analyzed with the jMRUI (http:// www.mrui.uab.es/mrui/_download) package. All MR spectroscopy data were zero-filled to 4096 data points and preprocessed with manual zero-order phase correction and $2-\mathrm{Hz}$ exponential apodization. The frequency of the water signal acquired at the onset was set to $4.7 \mathrm{ppm}$ and thereafter as the reference for all other spectral data. The residual water signal in water-suppressed scans was removed by using the Hanckel-Lanczos Singular Value Decomposition method. Spectroscopic data were Fourier-transformed for display and visual quality control purposes by using the Advanced Method for Accurate Robust and Efficient Spectral Fitting algorithm within the jMRUI package. Gaussian components were modeled in the frequency domain of the metabolites of interest, including choline, creatine, $\mathrm{N}$-acetylaspartate, and glutamate/glutamine. The chemical shifts (ie, frequencies) of the fitted metabolite peaks were reported to a precision of $0.001 \mathrm{ppm}$. Spectra were automatically discarded if the fitted line widths were $<1$ $\mathrm{Hz}$ or $>10 \mathrm{~Hz}$. During analysis of all spectra, with the tempera- 
ture-dependent water resonance nominally assigned to a 4.7-ppm chemical shift, the apparent "shift" is paradoxically imparted to the NAA peak, ostensibly bringing the NAA peak further toward high resonance by a range accounted for by the true water frequency shift.

Temperature-dependent changes in the relative frequency shift between $\mathrm{H}_{2} \mathrm{O}$ and NAA were analyzed by a simple linear regression model in SAS 9.3 (SAS Institute, Cary, North Carolina) for derivation of the temperature-dependent coefficient of the water-NAA frequency shift relative to temperature change. The computed correlation coefficient was compared with the reported/expected results from past studies and used for comparison of the 3 approaches detailed above. The slope of regression and intercept was determined, and the strength of correlation and accuracy were determined from the coefficient of determination $\left(R^{2}\right)$ and root-mean-square error, respectively. Statistical significance was established at $P<.05$.

\section{In Vivo Thermometry}

Two adult rhesus macaques were sourced from the Yerkes National Primate Research Center for noninvasive thermometry; rhesus macaques were selected for their phylogenetic similarities to humans in a parallel investigation of a nonhuman primate model of inducible stroke under investigation in our institution. All animal studies were performed under approval the Institutional Animal Care and Use Committee providing for the entirety of the experimental protocol. Briefly, irreversible endovascular occlusion was achieved by tandem deployable suture embolus delivered under superselective angiographic guidance to the right MCA by using a minimal interventional approach. ${ }^{31,32}$

Imaging was performed on a clinical $3 \mathrm{~T}$ whole-body system identical to that described for in vitro experiments and using a dedicated 8-channel phased array head coil for local signal reception. Baseline MR thermometry was performed with single- or multivoxel techniques, both using a paired approach acquiring separate non-water-suppressed and water-suppressed spectra (DIFF 1 approach). Single-voxel spectroscopy was acquired with the same parameters as described for the preceding in vitro experiments. Multivoxel spectroscopy was acquired with a 2D $8-\mathrm{cm}^{2}$ point-resolved spectroscopy sequence acquisition with the following sequence parameters: water spectrum: $\mathrm{TR}=1500 \mathrm{~ms}$; $\mathrm{TE}=30 \mathrm{~ms} ; \mathrm{NEX}=1$; metabolite spectrum: $\mathrm{TR}=1500 \mathrm{~ms} ; \mathrm{TE}=$ $30 \mathrm{~ms} ; \mathrm{NEX}=7$; acquisition time $=10$ minutes 31 seconds. Eight outer volume suppression bands were placed with specific effort to exclude areas of potential contamination. For both single- and multivoxel thermometry, baseline scanning was performed across 6 repetitions during a 3-hour scanning session of healthy adult macaques under $1 \%-1.5 \%$ isoflurane with continuous physiologic monitoring and maintenance of the rectal temperature at approximately $37^{\circ} \mathrm{C} .^{33}$ In separate experimental sessions, singleor multivoxel spectra were subsequently acquired at approximately 6 hours following endovascular MCA occlusion during a continuous MR imaging session focused on infarct evolution. Voxels were excluded on the basis of spectral corruption as defined for the in vitro methodology.

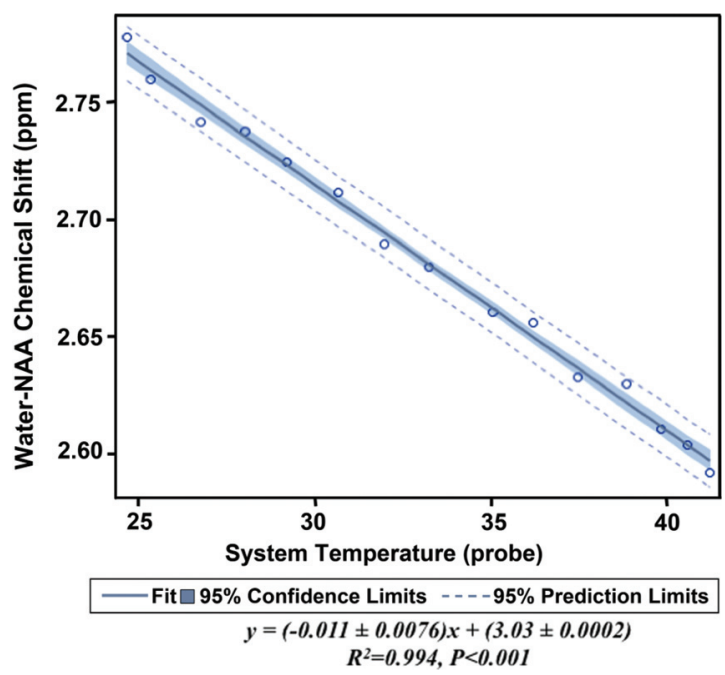

FIG 2. Linear regression from the DIFF 1 experiment. The $y$-axis represents the water-NAA chemical shift difference, plotted against continuous fiber optic measured temperatures from approximately 18 to $45^{\circ} \mathrm{C}$. The slope of regression, $0.01 \mathrm{ppm} /{ }^{\circ} \mathrm{C}$, is in agreement with the expected temperature dependency of the temperature-dependent proton chemical shift.

\section{RESULTS}

\section{Phantom Thermometry}

Before dynamic temperature measurements, minor fluctuations in phantom temperatures were observed due to equilibration and cold forced air within the scanner, varying between 18.8 and $19.0^{\circ} \mathrm{C}$; temperatures measured from $\Delta \delta_{\text {water-NAA }}$ varied from the probe temperature by $<0.3^{\circ} \mathrm{C}$ across 6 successive measures during this period. On temperature change with the flow-modulated phantom, $\Delta \delta_{\text {water-NAA }}$ demonstrated an expected linear decrease with increasing temperature for all 3 approaches. Phantom experiments for the 3 different methodologies demonstrated excellent agreement between probe and chemical shift thermometry.

For DIFF 1 (Fig 2), linear regression demonstrated the expected slope and intercept for the temperature-dependent proton chemical shift, $y=(-0.011 \pm 0.0076) x+(3.03 \pm 0.0002)\left(R^{2}=\right.$ 0.994 , root-mean-square error $=0.005, P<.001)$.

In an initial series of experiments using the DIFF 2 protocol, errors relating to apparent overestimation of temperatures were observed. Specifically, the process of repeated water suppression in successive spectra imparted distortions on the observed water peak, because the chemical shift selective suppression pulse is rendered increasingly askew during the course of temperature change. To address this error, in a subsequent experiment, we repeatedly refocused the center frequency on the shifting water resonance before acquisition of each DIFF 2 spectrum, eliminating the distortions (see "Discussion"). Using the DIFF 2 approach with the addition of this refocusing step, we observed excellent agreement with measured probe temperatures (Fig 3), $y=$ $(-0.010 \pm 0.0002) x+(3.00 \pm 0.0066)\left(R^{2}=0.996\right.$, root-meansquare error $=0.004, P<.001)$.

The DIFF 3 approach, proposed to further accelerate the existing methodologies, demonstrated excellent correlation with temperatures (Fig 4$)$, at $7 \times$ temporal resolution, $y=(-0.014 \pm$ $0.0001) x+(3.12 \pm 0.0016)\left(R^{2}=0.998\right.$, root-mean-square error $=0.00577, P<.001)$.

AJNR Am J Neuroradiol 36:1128-35 Jun 2015 www.ajnr.org 1131 


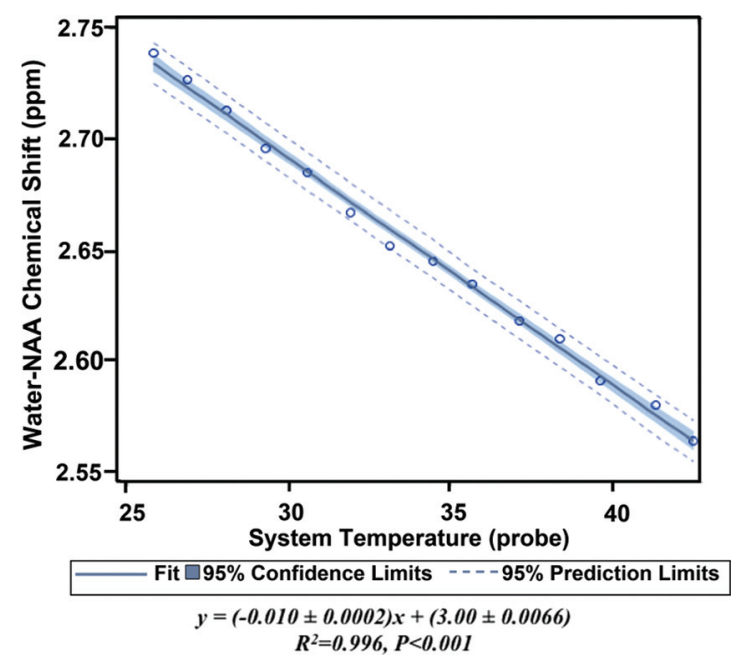

FIG 3. Linear regression from the DIFF 2 experiment. Similar correlation as noted in DIFF 1. By comparison with DIFF 1, approximately $15 \%-20 \%$ time savings is achieved by eliminating the paired approach used in Fig 2.

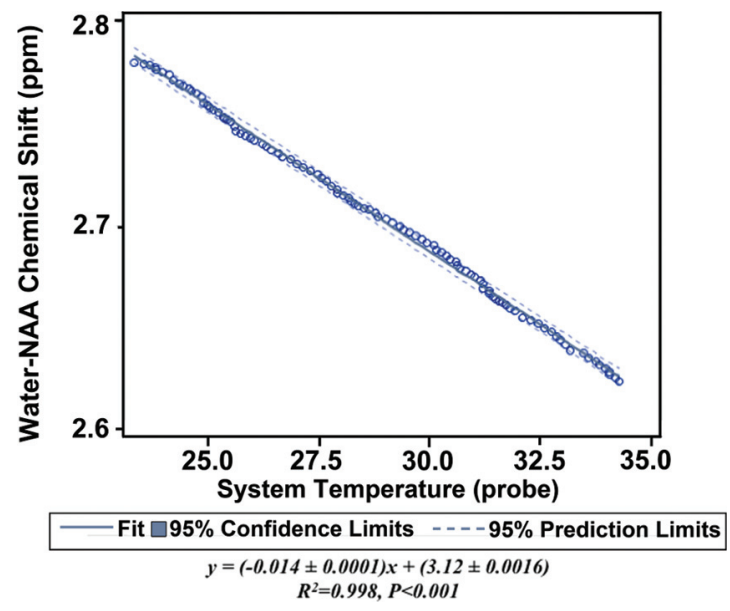

FIG 4. DIFF 3 versus temperature change. Excellent correlation is again noted between measured temperatures and the water-NAA chemical shift. Frequency shifts are measured from sequential, nonwater-suppressed spectra against an initial NAA reference frequency. Elimination of the repeated metabolite spectra in DIFF 1 and DIFF 2 permits increasing temporal resolution, with collection of 102 data points between approximately 18 and $35^{\circ} \mathrm{C}$, compared with $<15$ measures across a similar experimental session.

\section{In Vivo Thermometry}

Single-voxel spectroscopy (Fig 5) acquired across 6 repeated acquisitions during a single 3-hour imaging session demonstrated excellent spectral quality with fitted line widths all $<0.04 \mathrm{ppm}$ (approximately $5 \mathrm{~Hz}$ ) permitting reproducible determination of the water-NAA chemical shift difference. Mean and computed temperatures during maintenance of rectal temperatures at approximately $37^{\circ} \mathrm{C}$ were $36.94 \pm 0.22^{\circ} \mathrm{C}$.

Multivoxel spectroscopy (Fig 6) demonstrated similarly robust findings, though with corruption of peripheral-most voxels likely affected both by lipid contamination and chemical shift misregistration, despite meticulous outer volume suppression. An inner volume of $8 \times 4$ voxels was suitable for water-NAA chemical shift determination and demonstrated distinct zonality in computed temperatures, with relatively symmetric regions of

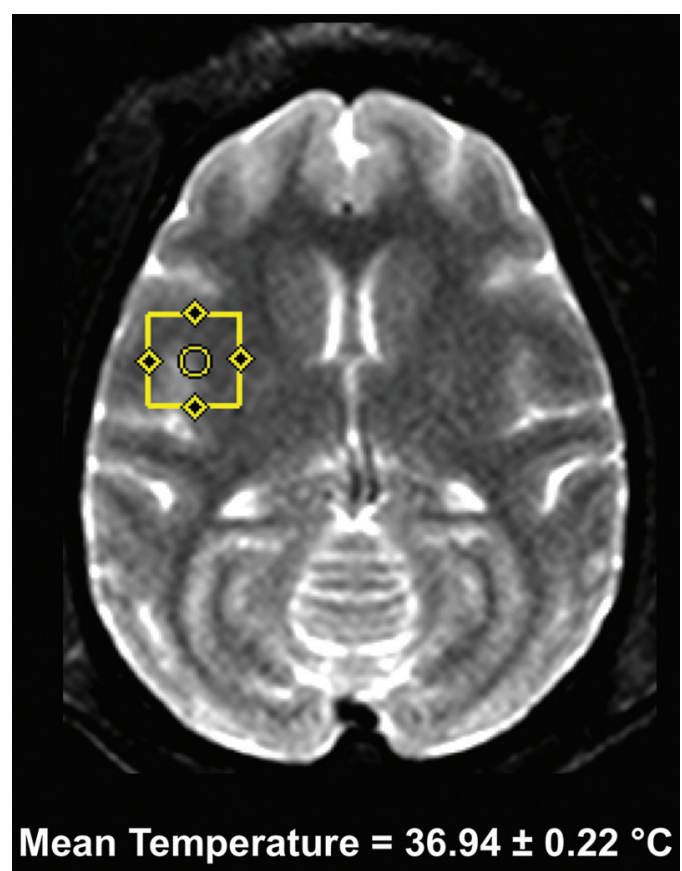

FIG 5. Single-voxel MR spectroscopy in a healthy nonhuman primate during physiologic monitoring under general anesthesia, with maintenance of rectal temperatures at approximately $37^{\circ} \mathrm{C}$. Six repeated MR spectroscopy scans were acquired from the right operculum by using a standard point-resolved spectroscopy sequence across an approximately 3-hour imaging session. Temperatures were computed from the temperature-dependent water-NAA chemical shift difference (see text), with mean computed temperatures of $36.94 \pm 0.22^{\circ} \mathrm{C}$. Spectral fitted full width at half maximum varies between 4 and $5 \mathrm{~Hz}$.

mean temperature variation ranging from a minimum of $36.3^{\circ} \mathrm{C}$ to a maximum of $39.3^{\circ} \mathrm{C}$.

Delayed single-voxel thermometry obtained 6 hours following right MCA occlusion (Fig 7) demonstrated slightly lower temperatures within the infarct territory compared with the contralateral normal hemisphere (36.66 versus $37.06^{\circ} \mathrm{C}$, respectively). Multivoxel spectroscopy 6 hours following induced ischemia (Fig 8) demonstrated evolution of the zonal thermal gradients observed during physiologic imaging conditions, also with generally lower temperatures throughout the infarct territory.

\section{DISCUSSION}

These findings establish the comparability of 3 competing approaches to PRF thermometry and introduce a fixed-NAA approach (DIFF 3), mitigating some of the speed limitations inherent to most PRF thermometry, without adverse effects on accuracy. Among the 2 commonly used approaches to chemical shift thermometry, denoted herein as DIFF 1 and DIFF 2, we observed excellent correlation for both, confirming that the conventional use of paired water and metabolite spectra (DIFF 1) could be supplanted by a more efficient single-scan approach (DIFF 2) as recently proposed. ${ }^{27}$ While such an approach offers improved temporal resolution without concern for interscan motion (ie, between the paired water and metabolite spectra), implementation is not without potential pitfalls. Specifically, simply acquiring successive partially water-suppressed spectra for $\Delta \delta_{\text {water-NAA }}$ may engender distortions of the observed water peak. The chemical shift selective suppression pulse intended for water 


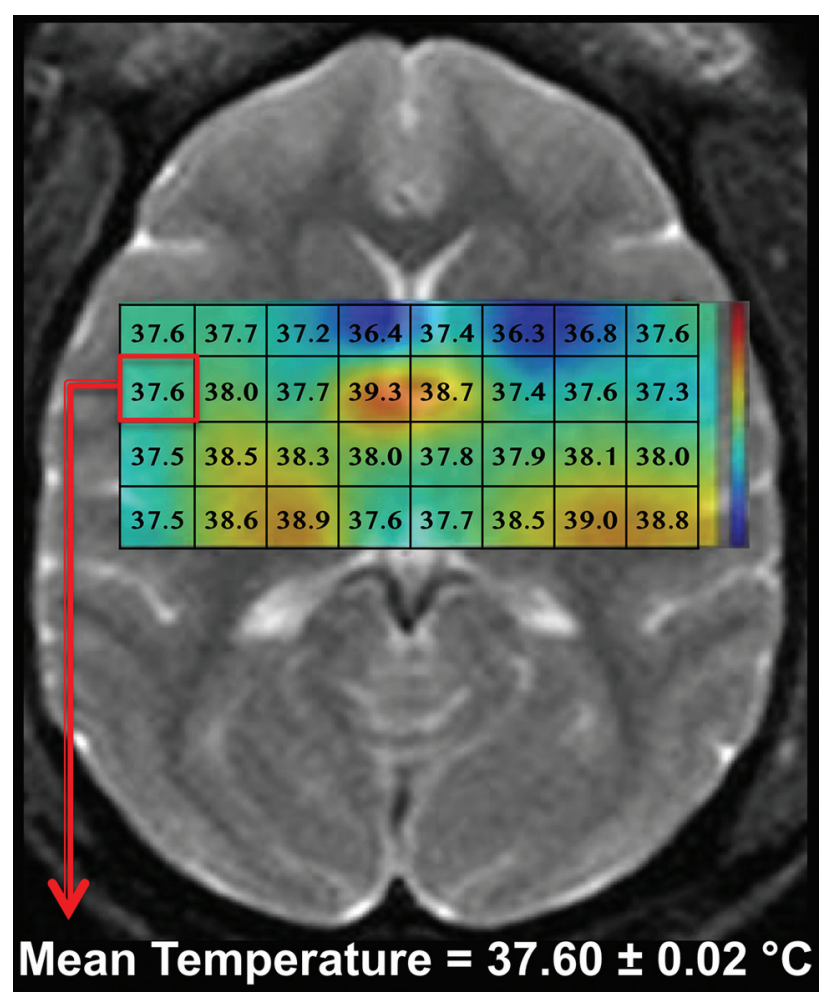

FIG 6. Multivoxel MR thermometry in a healthy nonhuman primate during physiologic monitoring under general anesthesia, with maintenance of rectal temperatures at approximately $37^{\circ} \mathrm{C}$. Six repeated MR spectroscopy scans were acquired by using 2D chemical shift imaging spectroscopy (see text) across an approximately 3-hour imaging session. Computed temperatures are derived from the water-NAA proton chemical shift and color thermal grid overlaid on the axial T2-weighted reference image for display purposes. A relatively symmetric, zonal distribution of temperatures is noted across repeated studies; a representative peripheral voxel demonstrates the repeatability of in vivo imaging, with mean computed temperatures of $37.6 \pm 0.02^{\circ} \mathrm{C}$. Spectral-fitted full widths at half maximum vary between 4 and $5 \mathrm{~Hz}$ across voxels.

saturation is rendered increasingly askew during the course of temperature change during heating/cooling, and the consequent distortions on the water peak could confound measurements. This error relates to the nominal assignment of the water resonance at $4.7 \mathrm{ppm}$ at the onset, albeit with an unaccounted for temperature-dependent frequency shift, which, during heating, moves downstream and increasingly off-resonance. The suppression pulse, now off-resonance, may deform the water peak during curve-fitting and increasingly so with greater temperature change.

The DIFF 3 protocol offers further enhancements to the temporal resolution achievable in either DIFF 1 or DIFF 2. The basis for this approach can be understood in the context of water resonance frequency taken in isolation; one may consider the possibility of acquiring simply a succession of high temporal resolution ( $\sim 40$-second) non-water-suppressed scans, from which the changes of water resonance frequency relative to baseline could be used to derive relative temperature change, vis-à-vis phase-contrast thermometry techniques in widespread use. Such an approach would, however, eliminate the primary advantage of $\Delta \delta_{\text {water-NAA }}$ thermometry, specifically, the "internally referenced" nature of PRF, which allows comparing the temperaturedependent water resonance against a static reference frequency such as NAA. The NAA reference frequency thus accounts for non-temperature-related field drifts and other sources of error as detailed in past reports. ${ }^{26}$ We thus proposed that accelerated PRF thermometry might instead be achieved by the acquisition of a baseline hydrogen metabolite spectrum for NAA assignment, followed, in close succession, by serial non-water-suppressed spectra. By taking advantage of a baseline reference frequency provided by the initial metabolite spectrum, one can observe the benefits of $\Delta \delta_{\text {water-NAA }}$ thermometry in a more efficient protocol and without detriment to accuracy. The benefits of $>7 \times$ data collection speed could offset potential shortcomings with this approach, and indeed DIFF 3 demonstrated accuracy comparable with that of DIFF 1 and DIFF 2 in this experimental protocol.

The 0.004 difference between slopes of regression for the DIFF 3 methodology cannot be readily explained and could relate to partially uncompensated field drifts during the experimental session. In this respect, the benefits of acceleration may be balanced by the potential for errors and therefore, lesser accuracy as a method approaching absolute thermometry. Alternatively, the NAA (or other non-hydrogen-bound metabolite signatures) could be sampled periodically relative to water scans, hence still providing some relative acceleration while potentially mitigating any unaccounted for frequency drift.

Despite promising results for all 3 approaches, it must be noted that $\Delta \delta_{\text {water-NAA }}$, while superior as an approximation of absolute thermometry compared with competing techniques, several inherent limitations exist for $\Delta \delta_{\text {water-NAA }}$ as an absolute thermometer. ${ }^{26}$ These relate principally to errors in the detection of the frequency of the signal and arise largely from inter- and intravoxel inhomogeneity; while errors from intervoxel inhomogeneities are considerably ameliorated by using an internal reference frequency such as NAA, intravoxel inhomogeneities may induce smearing of spectra and impart inaccuracies during estimation of resonance frequencies, particularly when SNR is limited. These and other limitations have been significantly addressed by the use of spectral estimation techniques, which prove valuable in achieving the frequency resolution necessary to make chemical shift thermometry clinically relevant.

In the present study, an aqueous brain cytosol model was selected for the temperature validation experiments, building on prior experimental models described by Corbett et al, ${ }^{27}$ and ensuring and facilitating a predictable, controlled temperature response during heating.

$\Delta \delta_{\text {water-NAA }}$ represents the only thermometry technique determined purely from the frequency of proton resonance, in contrast to other techniques derived from amplitudes. In this respect, robust thermometry can be achieved even in disorders wherein neuronal injury may lessen detectable NAA, as shown from in vivo stroke thermometry conducted by Corbett et al. ${ }^{27,30,34}$ Furthermore, unique to this technique is its tissue-type insensitivity, owing to the predictability of the proton chemical shift with changing temperatures, an attribute shared only with the other PRF technique, water phase-contrast thermometry. By comparison with phase-contrast techniques however, chemical shift thermometry can potentially be performed without baseline scanning, while allowing the correction of non-temperature-related changes in the proton phase such as susceptibility variations. ${ }^{26}$ 
We further acknowledge several limitations in the study design, including, primarily, those obstacles precluding validation of primate MR spectroscopy temperatures with absolute probe measurements. Resource and technical limitations complicate the collection of temporospatial cerebral thermal gradients in the nonhuman primate model, and insofar as the rectal temperatures reflect brain temperatures, they must be considered an approximation. Applications of MR thermometry in the setting of neurovascular ischemia have been explored preliminarily, including initial reports by Marshall et $\mathrm{al}^{25}$ and Karaszewski et al, ${ }^{23}$ operationally describing a theoretic formalism for the evolving temperature gradients observed in the setting of ischemia. Technical obstacles preclude absolute measurement of cerebral spatiotemporal gradients, motivated by the development of noninvasive approaches as described here. Corbett et $\mathrm{al}^{27}$ observed gradients of $0.5-1.0^{\circ} \mathrm{C}$ across the superficial $1 \mathrm{~cm}$ of the brain in a small

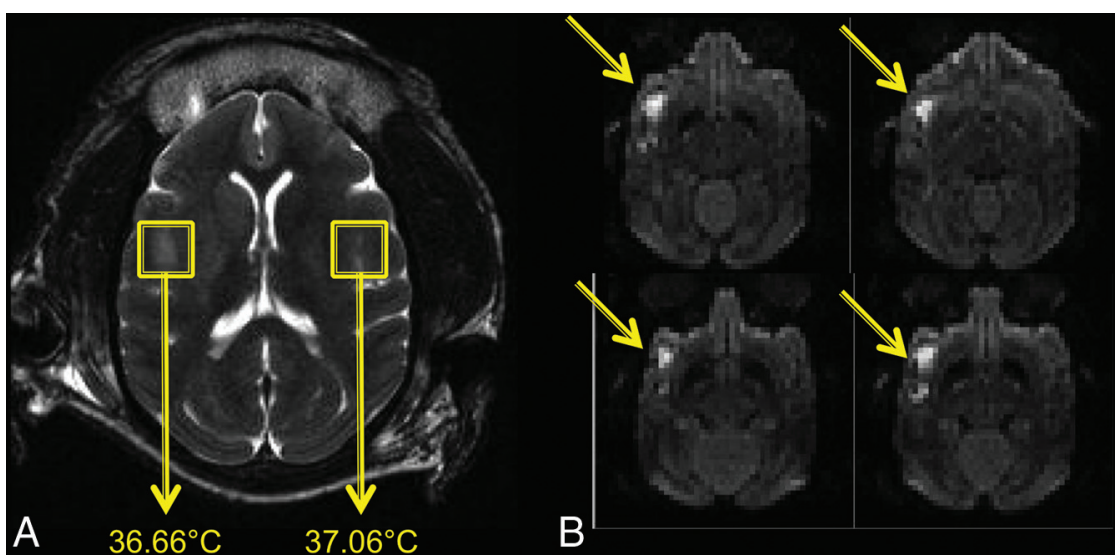

FIG 7. A, Single-voxel MR thermometry in an adult nonhuman primate 6 hours following endovascular MCA occlusion. Axial T2-weighted image (right) with symmetric 1.5-mL voxels placed in the bilateral opercular region. $B$, Area of infarction in the right hemisphere demonstrated on diffusion-weighted images (yellow arrows). Computed temperatures within the region of infarction are noted approaching $0.5^{\circ} \mathrm{C}$ less than those in the contralateral hemisphere. piglet stroke model and excellent agreement between localized probe and MR spectroscopy-derived temperatures. A theoretic framework of brain temperature regulation further highlights the potential for basal thermal gradients, relating to differences in blood flow, metabolism, and evaporative heat loss, with increasing variation predicted in functionally active regions. ${ }^{5}$

While the in vivo experiments herein represent only an initial experience with MR spectroscopy thermometry in the setting of a controlled, inducible primate stroke model, the diminished temperatures observed are consistent with predicted phenomena in their prior studies. Phantom experiments using multivoxel techniques were generally challenging in our model, due to the relatively small dimensions of the phantom, designed to ensure a uniform and predictable temperature response but limiting the use of multivoxel acquisition uncorrupted by the boundaries of the phantom. We have recently shown the comparability and lack of significant differences in multivoxel and single-voxel thermometry at static room temperatures; however, the phantom design in that protocol did not permit real-time probe temperature collection. ${ }^{35}$ We, however, maintain that application of the same spectral qualitycontrol measures as are in place for single-voxel spectroscopy and the excellent spectral quality in multivoxel experiments support the robustness of the spectra in those experiments. The potential impact of isoflurane on blood flow, metabolism, and thus potentially brain temperature could not be directly assessed within the experimental model in place. We aimed primarily to exhibit the feasibility of MR spectroscopy ther-

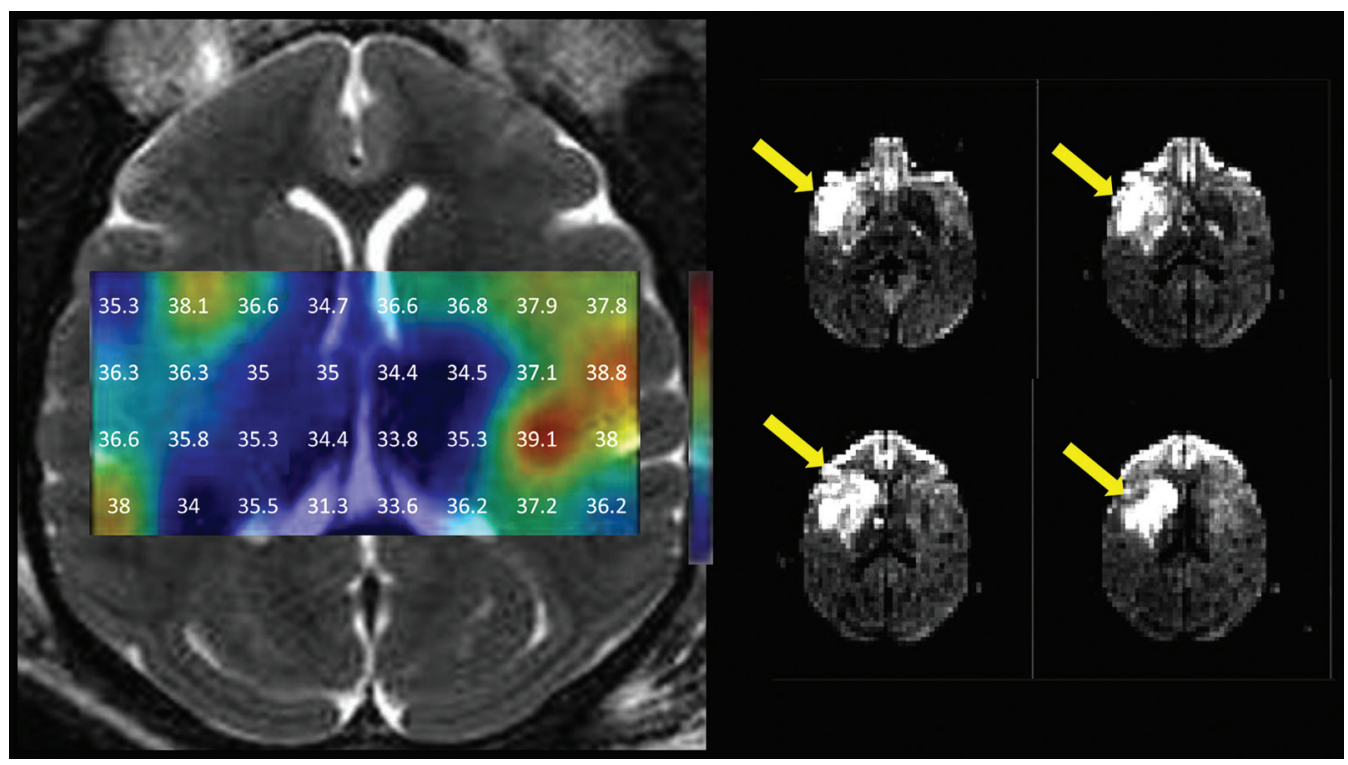

FIG 8. Multivoxel MR thermometry in an adult rhesus macaque obtained approximately 6 hours following endovascular MCA occlusion. Symmetric gradients observed under physiologic, preischemic conditions in Fig 6 were replaced by generally lower temperatures throughout the infarction territory of the right MCA seen on diffusion-weighted imaging (yellow arrows). Temperatures throughout the right hemispheric infarct territory generally diminish by comparison with the contralateral normal left hemisphere. 
mometry in the healthy and ischemic primate brain, and in this respect, the conditions of administration and monitoring and control of physiologic temperatures were preserved across experiments.

\section{CONCLUSIONS}

The potential applications of brain thermometry in normal physiology and disease are widespread, but characterization of cerebral temperatures has traditionally been limited by the costly and invasive nature of implantable thermometry systems.

Disclosures: Seena Dehkharghani-RELATED: Grant: Foundation of the American Society of Neuroradiology. ${ }^{*}$ Hui Mao-UNRELATED: Grants/Grants Pending: National Institutes of Health. * *Money paid to the institution.

\section{REFERENCES}

1. Baker MA. A brain-cooling system in mammals. Scientific American 1979;240:130-39

2. Brengelmann GL. Specialized brain cooling in humans? FASEB J 1993;7:1148-52; discussion 1152-53

3. Cabanac M. Selective brain cooling in humans: "fancy" or fact? FASEB J 1993;7:1143-46; discussion 1146-47

4. Hayward JN, Baker MA. Role of cerebral arterial blood in the regulation of brain temperature in the monkey. Am J Physiol 1968; 215:389-403

5. Sukstanskii AL, Yablonskiy DA. Theoretical model of temperature regulation in the brain during changes in functional activity. Proc Natl Acad Sci U S A 2006;103:12144-49

6. Busto R, Dietrich WD, Globus MY, et al. Small differences in intraischemic brain temperature critically determine the extent of ischemic neuronal injury. J Cereb Blood Flow Metab 1987;7:729-38

7. Erecinska M, Thoresen M, Silver IA. Effects of hypothermia on energy metabolism in mammalian central nervous system. J Cereb Blood Flow Metab 2003;23:513-30

8. Hammer MD, Krieger DW. Acute ischemic stroke: is there a role for hypothermia? Cleve Clin J Med 2002;69:770, 773-74, 776-77

9. Liu L, Yenari MA. Therapeutic hypothermia: neuroprotective mechanisms. Front Biosci 2007;12:816-25

10. Wolfe KB. Effect of hypothermia on cerebral damage resulting from cardiac arrest. Am J Cardiol 1960;6:809-12

11. Minamisawa $H$, Mellergard $P$, Smith ML, et al. Preservation of brain temperature during ischemia in rats. Stroke 1990;21:758-64

12. Minamisawa $\mathrm{H}$, Nordstrom $\mathrm{CH}$, Smith $\mathrm{ML}$, et al. The influence of mild body and brain hypothermia on ischemic brain damage. J Cereb Blood Flow Metab 1990;10:365-74

13. Schwab S, Spranger M, Aschoff A, et al. Brain temperature monitoring and modulation in patients with severe MCA infarction. Neurology 1997;48:762-67

14. Verlooy J, Heytens L, Veeckmans G, et al. Intracerebral temperature monitoring in severely head injured patients. Acta Neurochir (Wien) 1995; $134: 76-78$

15. Cady EB, D'Souza PC, Penrice J, et al. The estimation of local brain temperature by in vivo $1 \mathrm{H}$ magnetic resonance spectroscopy. Magn Reson Med 1995;33:862-67

16. Kiyatkin EA. Brain temperature fluctuations during physiological and pathological conditions. Eur J Appl Physiol 2007;101:3-17

17. Modi J, Bai HD, Menon BK, et al. Enhancing acute ischemic stroke interpretation with online aspects training. Can J Neurol Sci 2012; 39:112-14

18. Simon E. Tympanic temperature is not suited to indicate selective brain cooling in humans: a re-evaluation of the thermophysiological basics. Eur J Appl Physiol 2007;101:19-30

19. Soukup J, Rieger A, Holz C, et al. Temperature gradient between brain tissue and arterial blood mirrors the flow-metabolism relationship in uninjured brain: an experimental study. Acta Anaesthesiol Scand 2007;51:872-79

20. Rieke V, Butts Pauly K. MR thermometry. J Magn Reson Imaging 2008;27:376-90

21. Karaszewski B, Carpenter TK, Thomas RG, et al. Relationships between brain and body temperature, clinical and imaging outcomes after ischemic stroke. J Cereb Blood Flow Metab 2013;33:1083-89

22. Karaszewski B, Thomas RG, Dennis MS, et al. Temporal profile of body temperature in acute ischemic stroke: relation to stroke severity and outcome. BMC Neurol 2012;12:123

23. Karaszewski B, Wardlaw JM, Marshall I, et al. Measurement of brain temperature with magnetic resonance spectroscopy in acute ischemic stroke. Ann Neurol 2006;60:438-46

24. Karaszewski B, Wardlaw JM, Marshall I, et al. Early brain temperature elevation and anaerobic metabolism in human acute ischaemic stroke. Brain 2009;132:955-64

25. Marshall I, Karaszewski B, Wardlaw JM, et al. Measurement of regional brain temperature using proton spectroscopic imaging: validation and application to acute ischemic stroke. Magn Reson Imaging 2006;24:699-706

26. Kuroda K. Non-invasive MR thermography using the water proton chemical shift. Int J Hyperthermia 2005;21:547-60

27. Corbett RJ, Laptook AR, Tollefsbol G, et al. Validation of a noninvasive method to measure brain temperature in vivo using $1 \mathrm{H} \mathrm{NMR}$ spectroscopy. J Neurochem 1995;64:1224-30

28. Kuroda K, Mulkern RV, Oshio K, et al. Temperature mapping using the water proton chemical shift: self-referenced method with echoplanar spectroscopic imaging. Magn Reson Med 2000;43:220-25

29. Kuroda K, Suzuki Y, Ishihara Y, et al. Temperature mapping using water proton chemical shift obtained with 3D-MRSI: feasibility in vivo. Magn Reson Med 1996;35:20-29

30. Corbett R, Laptook A, Weatherall P. Noninvasive measurements of human brain temperature using volume-localized proton magnetic resonance spectroscopy. J Cereb Blood Flow Metab 1997;17:363-69

31. Zhang X, Tong F, Li CX, et al. A fast multiparameter MRI approach for acute stroke assessment on a 3T clinical scanner: preliminary results in a non-human primate model with transient ischemic occlusion. Quant Imaging Med Surg 2014;4:112-22

32. de Crespigny AJ, D’Arceuil HE, Maynard KI, et al. Acute studies of a new primate model of reversible middle cerebral artery occlusion. $J$ Stroke Cerebrovasc Dis 2005;14:80-87

33. Li CX, Patel S, Auerbach EJ, et al. Dose-dependent effect of isoflurane on regional cerebral blood flow in anesthetized macaque monkeys. Neurosci Lett 2013;541:58-62

34. Corbett RJ, Purdy PD, Laptook AR, et al. Noninvasive measurement of brain temperature after stroke. AJNR Am J Neuroradiol 1999;20:1851-57

35. Dehkharghani S, Wei L, Mao H, et al. Multivoxel proton spectroscopy for non-invasive MR thermometry: phantom comparison of PRESS and semiLASER-localized chemical shift imaging for temperature monitoring. In: Proceedings of the International Society for Magnetic Resonance in Medicine, Milan, Italy. May 10-16, 2014 